\title{
PHASE TRANSITION OF PRASEODYMIUM MONO-PNICTIDES UNDER HIGH PRESSURE
}

\author{
DINESH CHANDRA GUPTA \\ Condensed Matter Theory Group, School of Studies in Physics, Jiwaji University, GWALIOR - 474 011, INDIA \\ sosfizix@gmail.com;dcgfizix@gmail.com \\ GAJENDRA SINGH RAYPURIA \\ Department of Physics, Govt. K.R.G. P.G. Autonomous College, Gwalior-474 001, INDIA \\ gsraypuria@gmail.com
}

\begin{abstract}
The Phase transition and elastic properties of Praseodymium-monopnictides have been investigated under pressure by means of a modified charge-transfer potential model which incorporates the Coulomb screening due to the delocalization of f-electron of rare-earth atom leading to many-body interactions, along with Coulomb interaction, covalency effect and overlap repulsion extended up to second-nearest neighbours. These compunds undergo transition from $\mathrm{NaCl}$ structure to high pressure body-centered tetragonal (BCT) structure (distorted CsCl-type $\mathrm{P} 4 / \mathrm{mmm}$ ). The calculated values of cohesive energy, lattice constant, phase transition pressure, relative volume collapse. Present model explains the Cauchy's discrepancy correctly.
\end{abstract}

Keywords: Rare-earth compounds; High pressure phase transition; Equation of state.

\section{Introduction}

There is a growing experimental and theoretical interest in the study of rare earth (RE) mono-pnictides of praseodymium ( $\mathrm{PrX}: \mathrm{X}=\mathrm{P}, \mathrm{As}, \mathrm{Sb}, \mathrm{Bi}$ ) due to their intricate electronic structures and other diversed and unusual properties[1,2]. The interest in these materials has further increased after it was demonstrated that they can be grown epitaxially on III-IV semiconductors [3]. These monopnictides crystallize in $\mathrm{NaCl}$ crystal structure and can be studied within interaction potential theory which is relatively cheap and easy. From the theoretical point of view, the electronic properties of the RE compounds are mainly determined by the degree of localization of the $4 \mathrm{f}$ electrons, the treatment of which presents a considerable challenge to band theory. The PrX Compounds behave similarly to La- and Ce- compounds. X-ray diffraction on $\operatorname{PrX}(\mathrm{X}=\mathrm{P}$, $\mathrm{As}$ and $\mathrm{Sb}$ ) the $\mathrm{NaCl}$ has been performed at high pressures at room temperature [4,5]. The high-pressure phase of $\mathrm{PrX}$ is tetragonal (distorted CsCl-type P4/mmm). The powder Xray diffraction using the synchrotron radiation of cerium and praseodymium monopnictides [CeX and $\operatorname{PrX}(\mathrm{X}=\mathrm{P}, \mathrm{As}, \mathrm{Sb}$ and $\mathrm{Bi})]$ up to $42 \mathrm{GPa}$ at room temperature has been carried by Svane, et al.[7] They have also computed the electronic structures by self-interaction corrected local-spin density approximation. The high pressure 
structural behavior of the actinides and pnictides has also been reported by Benedict, et $a l$. [8,9]. The pressure induced structural phase transition occurs from B1 phase to bodycentered tetragonal structure, which may be viewed as a distorted B2 phase [10]. The isomorphous transformation in PrP occurs at around $26 \mathrm{GPa}$ with a volume collapse of $12.1 \%$ [10]. While in PrAs, PrSb and PrBi it occurs at with transition pressures of 27, 13 and $14 \mathrm{GPa}$ [11-13] respectively. Varshney et al. the models used by them are basic for explaining the crystal properties but they exclude Coulomb screening effect due to manybody interactions in the expressions, which play an important role in defining the crystal properties of heavy-earth compounds (HECs) and in particular, their expressions of elastic moduli are not correct. Their expressions actually lead one to $\mathrm{C}_{12}=\mathrm{C}_{44}$ while their results have shown $\mathrm{C}_{12} \neq \mathrm{C}_{44}$ which is contradictory and hence their approach is not realistic qualitatively and quantitatively and the results unreliable. To study the high pressure behaviour of these compounds, we have developed an improved potential model which incorporates proper crystal interactions viz., long-range (LR) Coulomb and Coulomb screening due to the delocalization of electron of the rare-earth atom (chargetransfer or many-body interaction (CTI or MBI) [14]) modified by covalency effect [15] and short-range (SR) repulsion extended up to second-nearest neighbours. The expressions derived from the present model are capable of explaining the crystal properties correctly. Therefore, we have used the modified expressions of the secondorder elastic constants (SOECs), their combinations, Cauchy's discrepancy, third-order elastic constants (TOECs) and first- and second-order pressure derivatives of SOECs for present study as reported earlier [17-19]. The corrected part has been underlined in these expressions. In this next section, we have described in detail the potential model and followed by methodology used to compute the model parameters. The results have been discussed in section 4 .

\section{Potential Model}

The relative stability of the two competitive phases, i.e., B1 and $\mathrm{B} 2$ has been studied by minimizing the Gibbs free (GF) energy ( $\mathrm{G}=\mathrm{U}+\mathrm{PV}-\mathrm{TS})$ at zero and high pressures using iterative self-consistent method. At phase transition pressure $\left(\mathrm{P}_{\mathrm{T}}\right)$ both the phases coexist. The cohesive energy is expressed as

$\mathrm{U}(\mathrm{r})=\mathrm{U}_{\mathrm{Coul}}+\mathrm{U}_{\mathrm{CTI}}+\mathrm{U}_{\mathrm{Cov}}+\mathrm{U}_{\mathrm{HF}}$

Or $\quad U(r)=-\frac{\alpha_{M} e^{2} Z_{m}{ }^{2}}{r}+b \sum_{i j} \beta_{i j} \exp \left(\frac{r_{i}+r_{j}-r_{i j}}{\rho}\right) \quad$ with $Z_{m}{ }^{2}=Z\left(Z+2 n f_{m}(r)\right)$

The various terms in above equation have their usual meaning and are well described elsewhere [16]. The value of CTI parameter depends on the nearest neighbour (nn) distance and can be estimated from the analytic expression $f_{C T I}(r)=f_{o} e^{-r / \rho}$ with $f_{o}$ as CTI parameter, here $\rho$, is range parameter. The values of the model parameters, viz. b, $\rho$ and $\mathrm{f}_{\mathrm{m}}(\mathrm{r})$ have been obtained from the equilibrium condition and compressibility or bulk modulus $\left(\mathrm{B}_{\mathrm{T}}\right)$ as $\mathrm{dU} /\left.\mathrm{dr}\right|_{\mathrm{r}=\mathrm{ro}}$ and $\mathrm{d}^{2} \mathrm{U} / \mathrm{dr}^{2}=9 \mathrm{KrB}_{\mathrm{T}}$ with $\mathrm{K}\left(=\mathrm{V} / \mathrm{r}^{3}\right)$ as structure constant. 
The potential has been used to derive the expressions of the elastic moduli, their combinations and pressure derivatives on the lines suggested by [14]. Here, it is interesting to note that the corrected term as underlined in these expressions [16] were ignored by Gour, et al. [17] and Varshney, et al. [18] which upon further examination actually lead one to $\mathrm{C}_{12}=\mathrm{C}_{44}$ while our analysis naturally expresses the Cauchy's discrepancy $\left(\mathrm{C}_{12}-\mathrm{C}_{44} \neq 0\right)$ in terms of CTI parameter. The relation for Cauchy's discrepancy from the present derivations comes out to be $\mathrm{C}_{12}-\mathrm{C}_{44}=9.3204 \mathrm{Z} \mathrm{e} \mathrm{e}^{2}$ af' $(\mathrm{r}) / 4 \mathrm{a}^{4}$ for $\mathrm{NaCl}$ structure.

The expressions for TOECs, first- and second-order pressure derivatives of SOECs have also been derived on the lines given by [14]. The higher derivatives of the CTI parameter $\mathrm{f}(\mathrm{r})$, i.e., af '(r), $\mathrm{a}^{2} \mathrm{f}$ "( $(\mathrm{r})$ and $\mathrm{a}^{3} \mathrm{f}$ "'(r) have been evaluated from the analytical expression $f(r)=f_{0} e^{-r / \rho}$. The method of determination of the parameters has been described in the following section.

\section{Computation of Model Parameters}

The MCTP model has low parametric nature and hence consists of only three parameters, namely, hardness (b), range $(\rho)$ and modified CTI parameter $f_{m}(r)$. The values of these model parameters have been calculated from the self-consistent method using the compressibility or bulk modulus and equilibrium condition [16] and experimental values of lattice constant to obtain the optimized values of cohesive energy and volume which in term gives the values of model parameters. The values of the input data along with the computed model parameters are listed in Table 1.

\section{Results and Discussions}

In order to check the relative stability of the two competitive phases, we have minimized the lattice energies in both real $\mathrm{B} 1\left(\mathrm{G}_{\mathrm{B} 1}\left(\mathrm{r}_{\mathrm{B} 1}\right)=\mathrm{U}_{\mathrm{B} 1}\left(\mathrm{r}_{\mathrm{B} 1}\right)+\mathrm{PV}_{\mathrm{B} 1}-\mathrm{TS}\right)$ and high pressure phase $\mathrm{B} 2\left(\mathrm{G}_{\mathrm{B} 2}\left(\mathrm{r}_{\mathrm{B} 2}\right)=\mathrm{U}_{\mathrm{B} 2}\left(\mathrm{r}_{\mathrm{B} 2}\right)+\mathrm{PV}_{\mathrm{B} 2}-\mathrm{TS}\right)$ phases at ambient conditions by selfconsistent technique to obtain the consistent value of lattice constant at ambient and high pressure conditions. To check the relative stability, we have minimized the lattice energies in both B1 and B2 phases at ambient conditions corresponding to equilibrium

Table 1: Input data $\mathrm{r}_{0}$ (in $10^{-10} \mathrm{~m}$ ), $\mathrm{B}_{\mathrm{T}}$ (in $\mathrm{GPa}$ ) and model parameters $\mathrm{b}$ (in $10^{-19} \mathrm{~J}$ ), $\rho$ (in $10^{-10} \mathrm{~m}$ ) and $\mathrm{f}_{0}$ (unit free) for Prmonopnictides.

\begin{tabular}{lcc|ccc}
\hline \multirow{2}{*}{ Solids } & \multicolumn{2}{c|}{ Input data } & \multicolumn{3}{c}{ Model parameters } \\
\cline { 2 - 6 } & $\mathbf{r}_{\mathbf{o}}$ & $\mathbf{B}_{\mathbf{T}}$ & $\mathbf{b}$ & $\boldsymbol{\rho}$ & $\mathbf{f}_{\mathbf{o}}$ \\
\hline PrP & $2.950^{\mathrm{a}}$ & $74 \pm 2^{\mathrm{a}}$ & 156.88 & 0.321 & 958.17 \\
PrAs & $3.000^{\mathrm{a}}$ & $100 \pm 7^{\mathrm{a}}$ & 148.21 & 0.312 & 1455.00 \\
PrSb & $3.175^{\mathrm{b}}$ & $44 \pm 5^{\text {a }}$ & 215.55 & 0.338 & 1603.99 \\
PrBi & $3.230^{\text {a }}$ & $40 \pm 5^{\text {a }}$ & 180.87 & 0.368 & 810.14 \\
\hline
\end{tabular}

${ }^{\mathrm{a}}$ Ref. [1]; ${ }^{\mathrm{b}}$ Ref. [4] 
interionic separations $\mathrm{r}_{\mathrm{B} 1}\left(\mathrm{r}_{\mathrm{B} 2}\right)$ using the MCTP model parameters listed in Table 1. The corresponding Gibbs free $(\mathrm{GF})$ energies $\mathrm{G}_{\mathrm{B} 1}\left(\mathrm{r}_{\mathrm{B} 1}\right)$ and $\mathrm{G}_{\mathrm{B} 2}\left(\mathrm{r}_{\mathrm{B} 2}\right)$ at difference pressures have also been computed and plotted in figure 1 . The pressure at which these GF energies cut each other have been marked by arrows as the phase transition pressure $\left(\mathrm{P}_{\mathrm{T}}\right)$ in these figures and the values of $\mathrm{P}_{\mathrm{T}}$ have also been reported in Table 2 along with experimental and other theoretical values. The GF energy difference $\Delta \mathrm{G}\left[=\mathrm{G}_{\mathrm{B} 2}\left(\mathrm{r}_{\mathrm{B} 2}\right)-\mathrm{G}_{\mathrm{B} 1}\left(\mathrm{r}_{\mathrm{B} 1}\right)\right]$ has also been computed as a function of pressure. It is seen that the value of $\Delta \mathrm{G}$ at zero pressure is positive in all the cases which is the requirement for correct stability of the competitive phases.

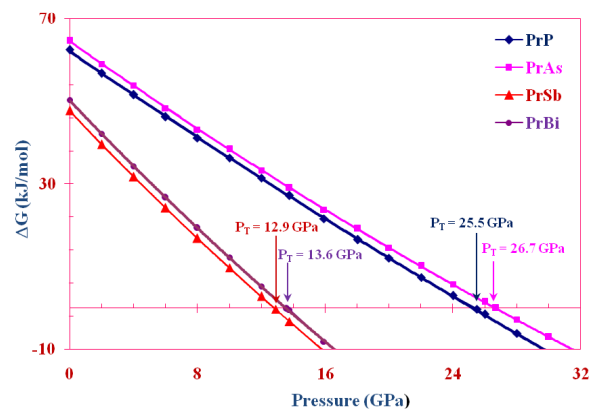

Fig. 1 Variation of Gibbs free energy in B1 and B2 phase with pressure for Pr-monopnictides.

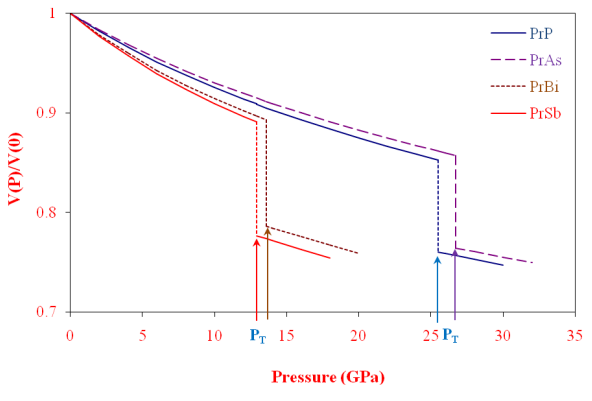

Fig. 2 Variation of reduced volume $(\mathrm{V}(\mathrm{P}) / \mathrm{V}(0))$ with pressure for Pr-monopnictides.

Thus the present model predicts correctly that all the Pr-monopnictides are thermodynamically and mechanically stable in $\mathrm{B} 1$ phase at ambient conditions. The value of $G_{B 1}$ remains minimum up to just before $P_{T}$ and becomes equal to $G_{B 2}$ at $P_{T}$ exhibiting the coexistence of both the phases at this pressure. Beyond this pressure the energy in $\mathrm{B} 2$ phase becomes more negative as compared to that in $\mathrm{B} 1$ phase predicting thereby that the high pressure B2 phase is now thermodynamically and mechanically stable as compared to $\mathrm{B} 1$ phase. It may be seen from this table that the values of $\mathrm{P}_{\mathrm{T}}$ computed by us are in good agreement with experimental data [13] and better than those obtained by earlier theoretical workers $[19,20]$.

These self-consistent values of lattice constant have been used to compute the reduced volume $(\mathrm{V}(\mathrm{P}) / \mathrm{V}(0))$ at different pressure in both $\mathrm{B} 1$ and $\mathrm{B} 2$ phases. The values of reduced volume at different pressures in both the phases have been plotted in figure 2 to obtain the equation of state (EOS) and in consequence the $\%$ volume collapse $\left(\mathrm{V}\left(\mathrm{P}_{\mathrm{T}}\right) / \mathrm{V}(0)\right)$ at $\mathrm{P}_{\mathrm{T}}$ for these compounds. These values along with $\mathrm{P}_{\mathrm{T}}$ have also been reported in Table 2. Our computed values of $\mathrm{P}_{\mathrm{T}}(=25.5,26.7,12.9$ and $13.6 \mathrm{GPa})$ and \% volume collapse $(=9.3,9.3,11.4$ and 9.6) are in good agreement with the experimental data [13] and better than those obtained by earlier workers [19,20] for PrP, PrAs, PrSb and $\mathrm{PrBi}$, respectively. 
Table 2: Calculated values of interionic separation $\left(r_{B 1}\right.$ and $r_{B 2}$ in $\left.10^{-10} \mathrm{~m}\right)$, Gibbs free energy $\left(\mathrm{G}_{\mathrm{B} 1}\right)$ and its difference $\Delta \mathrm{G}$ (in $\left.\mathrm{kJ} / \mathrm{mol}\right)$, phase transition pressure $\left(\mathrm{P}_{\mathrm{T}}\right.$ in $\left.\mathrm{GPa}\right)$ and $\%$ volume collapse $\left(\mathrm{V}\left(\mathrm{P}_{\mathrm{T}}\right) / \mathrm{V}(0)\right)$ for Pr-monopnictides.

\begin{tabular}{cccccccc}
\hline Solids & $\mathbf{r}_{\mathbf{B} 1}$ & $\mathbf{r}_{\mathbf{B} 2}$ & $\mathbf{G}_{\mathbf{B} 1}$ & $\Delta \mathbf{G}$ & $\mathbf{P}_{\mathbf{T}}$ & $\% \Delta \mathbf{V}\left(\mathbf{P}_{\mathbf{T}}\right) / \mathbf{V}(\mathbf{0})$ & References \\
\hline \multirow{4}{*}{ PrP } & 2.94 & 3.13 & -2177.3 & 63.1 & 25.5 & 9.3 & Present \\
& 2.95 & - & - & - & 26 & - & Expt. [13] \\
& - & - & - & - & 25 & 8.1 & Others [19] \\
& - & - & - & - & 16 & 12.1 & Others [20] \\
\hline \multirow{4}{*}{ PrAs } & 2.98 & 3.19 & -2207.1 & 64.6 & 26.7 & 9.3 & Present \\
& 3.00 & - & - & - & 27 & 9.0 & Expt. [13] \\
& 3.01 & 3.18 & -2788.5 & 93.3 & 26 & 8.6 & Others [19] \\
& - & - & - & - & 12 & - & Others [20] \\
\hline \multirow{4}{*}{ PrSb } & 3.21 & 3.32 & -2023.7 & 47.7 & 12.9 & 11.4 & Present \\
& 3.19 & - & - & - & 13 & 11.0 & Expt. [13] \\
& 3.20 & 3.37 & -1882.8 & 60.6 & 13 & 9.7 & Others [19] \\
& - & - & - & - & 8 & 8.9 & Others [20] \\
\hline \multirow{4}{*}{ PrBi } & 3.26 & 3.40 & -2011.2 & 50.3 & 13.6 & 9.6 & Present \\
& 3.23 & - & - & - & 14 & 8.0 & Expt. [13] \\
& 3.24 & 3.42 & -1816.2 & 61.2 & 13.4 & 9.0 & Others [19] \\
& - & - & - & - & - & 8.7 & Others [20] \\
\hline
\end{tabular}

\subsection{Elastic properties}

To analyses the mechanical strength of these compounds, we have computed SOECs and their combinations, Cauchy's discrepancy and Poisson's ratio $(\sigma)$ at ambient condition as well as at high pressures.

\section{Acknowledgment}

The authors are thankful to and UGC and DST, New Delhi for financial support.

\section{References}

1. F. Hullinger, in Hand Book on the Physics and Chemistry of Rare Earths, vol. 4, K.A. Gschneidner Jr and L. Eyring, eds., North Holland Physics Publishing, New York, 1979.

2. G. Pagare, V. Srivastava, S.P. Sanyal, and R.K. Singh, Phys. Stat. Sol. (b) 241 (2004), 3197.

3. Allen S J, Brehmer D and Palmstrom C J Rare Earth Doped Semiconductors vol 301, ed G S Pomrenka, P B Klein and D W Langer (Pittsburg, PA: Materials Research Society) 1993.

4. J.M. Leger, K. Oki, J. Rossat-Mignod, J. Phys. C 17 (1984) 4935.

5. J.M. Leger, K. Oki, J. Rossat-Mignod, O. Vogt, J. Phys. 46 (1985) 889.

6. A. Svane, Z. Szotek, W.M. Temmerman, and H. Winter, Solid State Commun. 102 (1997) 477.

7. A. Svane, Z. Szotek, W.M. Temmerman, J. Laegsgaard, and H. Winter, J. Phys.: Condens. Matter 10 (1998), 5325.

8. U. Benedict, S. Dabos-Seignon, J.P. Dancausse, M. Gensini, G. Gering, S. Heathman, H. Luo, J. Staun Olsen, L. Gerward, and R.G. Haire, J. Alloys, Compd. 181 (1992),12.

9. U. Benedict, J. Alloys. Compd. 223 (1995), 225.

10. T. Adachi, I. Shirotani, J. Hayashi, and O. Shimomura,Phys. Lett. A 250 (1998), 393.

11. I. Shirotani, K. Yamanashi, J. Hayashi, Y. Tanaka, and N. Ishimatsu, J. Phys.: Condens. Matter 13 (2001), 1946. 
12. J. Hayashi, I. Shirotani, Y. Tanaka, T. Adachi, O. Shimomura, and T. Kikegawa, Solid State Commun. 114 (2000), 565.

13. I. Shirotani, J. Hayashi, K. Yamanashi, K. Hirano, T. Adachi, N. Ishimatsu, O. Shimomura, and T. Kikegawa, Physica B 334 (2003), 174.

14. R.K. Singh, Phys. Rep. 85 (1982), 259.

15. K. Motida, J. Phys. Soc. Japan. 55 (1986), 1636.

16. D.C. Gupta and K.C. Singh, Phase transitions 83 (2010), 182; J. Alloys Compd. 499 (2010), 90; IL Nuovo Cimento D9 (1987), 1253.

17. A. Gour, S. Singh and R.K. Singh, Central Europ. J. Phys., 5 (2007), 576.

18. D. Varshney, N. Kaurav, P. Sharma, S. Shah and R.K. Singh, Phys. Stat. Sol. (b) 241 (2004), 3179; J. Alloys Compd. 495 (2010) 23. [and references therein].

19. P. Soni, G. Pagare, V. Shrivastava, S.P. Sanyal, Phase Transition 82, (2009), 530.

20. G. Vaitheeswaran, L. Petit, A. Svane, V. Kanchana, M. Rajagopalan, J. Phys.: Condens. Matter 16 (2004), 4440.R. Loren and D. B. Benson, J. Comput. System Sci. 27, 400 (1983). 